\title{
To investigate the relation of hypertension and anthropometric measurement among elderly in Malaysia.
}

\begin{abstract}
This study aimed to determine the association between anthropometric measurement and dyslipidemia and their association with hypertension among older people. A cross sectional study was done among the residents in two old folks homes (Rumah Seri Kenangan Cheras and Little Sisters of the Poor) in urban Malaysia using a pre-tested guided questionnaire. Measurements taken were the blood pressure level, height and weight measurement and lipid profile. There were 92 respondents participated in the study. The prevalence of hypertension was $51.1 \%$. The study found significant association between anthropometric measurement and hypertension although there was no difference in waist hip ratio measurement among hypertensive and normotensive individuals. Level of total cholesterol, LDL-C and triglycerides were significantly higher among hypertensive individuals compared to normotensive individuals. The prevalence rate of hypertension among residents in this study is higher compared to the national prevalence rate among the older adults. Appropriate invention programmes should be reinforced to reduce the complications of hypertension especially among institutionalized elderly.
\end{abstract}

Keyword: Aging; Anthropometric; Hypertension; Lipids 